\title{
CORRESPONDENCIAS LÉXICAS EN EL ESPAÑOL DE COSTA RICA: EL CHICHEME
}

\author{
Lexical correspondences in Costa Rican Spanish: chicheme
}

\author{
Alberto Barahona Novoa ${ }^{1}$
}

\begin{abstract}
RESUMEN
La variación y dispersión geográfica del léxico constituye un indicio para conocer la formación de zonas dialectales y tradiciones culturales compartidas por comunidades de habla. En este artículo, se examina el caso de la palabra chicheme para evidenciar los nexos lingüísticos y culturales entre la región noroeste, el Caribe y la zona sur de Costa Rica.

Palabas clave: dialectología, español de Costa Rica, gastronomía, chicheme, zonas dialectales.

ABSTRACT

The geographical variation and dispersion of the lexicon is an indication of the formation of dialect areas and cultural traditions shared by speaking communities. This article examines the case of the word chicheme for evidence of the linguistic and cultural links between the northwest region, the Caribbean and the southern zone of Costa Rica.
\end{abstract}

Key words: dialectology, Costa Rican Spanish, gastronomy, chicheme, dialect areas.

\section{Introducción}

La relación entre lengua y cultura se manifiesta de muchas maneras en la configuración espacial de las variedades lingüísticas. Por ello, no es de extrañar que los alimentos y bebidas de la dieta de una comunidad sean un escenario propicio para apreciar diferencias geolectales, máxime que la gastronomía está íntimamente ligada a la producción agrícola de las zonas geográficas de un país. En el caso de Costa Rica, las regiones del noroeste del país y del Valle Central muestran diferencias en las características geográficas, lingüísticas, de producción agronómica y en las costumbres alimentarias.

\footnotetext{
${ }^{1}$ Universidad de Costa Rica. Profesor catedrático del Departamento de la Escuela de Filología, Lingüística y Literatura. Costa Rica.

Correo electrónico: jose.barahona@ucr.ac.cr

Recepción: 29-08-2017 Aceptación: 15-01-2018

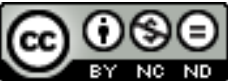

Esta obra está bajo una licencia de Creative Commons Reconocimiento-NoComercial-SinObraDerivada 4.0 Internacional
} 
En el plano lingüístico, esta diversidad es fácil de apreciar si se entra a considerar la variación de los diferentes niveles de la lengua, en especial el léxico-semántico. Para evidenciar esta diferenciación léxico-semántica entre estas dos zonas dialectales costarricenses, me serviré del examen del uso de la voz chicheme y su extensión por la geografía dialectal en nuestro país. Este vocablo se documenta en varias zonas, aunque el referente experimenta adaptaciones en la preparación de la bebida obtenida a partir de los granos de maíz. Ahora bien, la conservación de la receta -pese a las adaptaciones en su elaboración- y la retención del término es una evidencia de los posibles parentescos entre los dialectos del español en Costa Rica; por su parte, su desconocimiento apunta a la existencia de otro dialecto y una tradición cultural diferenciada entre zonas nacionales. En fin, se pretende destacar cómo la existencia de una isoglosa de un término gastronómico dibuja o visibiliza las diferencias dialectales.

Antes de pasar a conocer detalles sobre el chicheme, bebida presente en los hábitos alimentarios de varias zonas geográficas costarricenses, es necesario realizar algunas apreciaciones teóricas sobre la variación léxica, así como las fuentes bibliográficas que la atestiguan.

\section{La variación léxica}

De acuerdo con Moreno Fernández (1998), la variación de las unidades lingüísticas -sin importar el nivel al que pertenezcan- puede deberse a factores meramente lingüísticos o bien extralingüísticos. Por ello, establece la siguiente hipótesis:

Es posible proponer una diferenciación, al menos como hipótesis de trabajo, entre los niveles de la lengua según la naturaleza de las variables explicativas que en ellos suelen incidir: mientras la variación fonético-fonológica y la de tipo morfológico y funcional se ven determinadas frecuentemente por factores lingüísticos y extralinguiísticos, la variación categorial y posicional (sintáctica) se ve explicada mayoritariamente por factores lingüísticos y la variación léxica por factores extralingüísticos (Moreno Fernández, 1998, pp. 30-31). 
Aceptada esta asociación entre factores y niveles, cabe preguntarse sobre cuáles pueden ser los condicionamientos extralingüísticos; pues bien, podría pensarse en los rasgos sociológicos, situaciones históricas, factores ambientales, creencias o actitudes de las comunidades de habla, entre otros factores. En el caso que se comentará, definitivamente los hábitos culturales asociados a la alimentación, los productos de la tierra y las migraciones han influido en la configuración léxica de las variedades dialectales costarricenses.

En primer lugar, el cultivo del maíz y, en especial, el consumo de una variedad de color morado (maíz pujagua ${ }^{2}$ ) crea una realidad cultural diferente; de ahí surgen palabras y denominaciones de bebidas que la evidencia, creando, de esta manera, un inventario léxico diferenciado entre las variedades lingüísticas. Además, en la historia de nuestro país, la actividad económica ha obligado a que grupos humanos tuvieran que migrar a nuevas zonas y, con esto, la expansión de sus costumbres lingüísticas a otros escenarios. Estos factores extralingüísticos, sobre todo, han influido en el cambio lingüístico y la configuración de las zonas dialectales del español costarricense.

Ahora bien, la variación en el nivel léxico puede presentarse de varias maneras. Moreno Fernández (1998, p. 29) expone que pueden presentarse formas léxicas divergentes que pueden verse neutralizadas en su contenido semántico, sobre todo en el discurso. A su juicio, la sociolinguiística ha tenido que aceptar la existencia de la sinonimia, a sabiendas de que siempre intervienen factores como las intenciones comunicativas o las intenciones de estilo. En este sentido, se podría decir que se utilizan formas diferentes para comunicar lo mismo.

No obstante, la variación léxica también se percibe en el uso de formas iguales con material semántico dispar o con modificaciones al contenido original, propiciadas por los nuevos entornos culturales y geofísicos de las comunidades de habla. Es decir, se puede contar con formas únicas que, por razones extralingüísticas, han modificado, ampliado o mermado su contenido léxico. El vocablo por comentar se asume desde esta perspectiva de análisis.

\footnotetext{
2 Arturo Agüero (1996, p. 170), en su Diccionario de costarriqueñismos, sostiene que la etimología de pujagua "viene del náhuatl poxauac o puxauac, blando, esponjoso". Es decir, la voz responde a la macrozona dialectal mesoamericana.
} 
Estas posibilidades de apreciar la diferenciación léxica son distinguidas, desde hace mucho, en los estudios lingüísticos. Se ha convenido en llamar correspondencias léxicas a las formas únicas que pueden referir a contenidos semánticos divergentes, en la estructura externa de relaciones entre dialectos de una lengua histórica. Esta nomenclatura es la que conviene adoptar para el estudio de la variación de la voz chicheme, objeto de este artículo.

Estas dos formas de variación actuaron en la conformación del español en América. Es harto conocido que muchas voces experimentaron adaptaciones a la nueva realidad americana. En el campo de la gastronomía, baste mencionar el marinerismo mazamorra que, en la culinaria hispánica, correspondía a las migas de galletas con las cuales se realizaba una sopa cuando escaseaban los víveres en una embarcación; por su parte, en América, se resemantiza y se convierte en un especie de atol preparado a base de maíz, con preparaciones muy diversas en toda América.

\section{Zonas dialectales costarricenses y migraciones}

\subsection{Zonas dialectales}

Desde temprano, los estudios dialectológicos han distinguido dos variedades en el español de Costa Rica, es decir: la variedad vallecentraleña - para otros habla cartaga- y la variedad del noroeste o habla guanacasteca. La existencia de estas zonas dialectales está en consonancia con el proceso de poblamiento de nuestro país, en los albores de nuestra historia. Los demógrafos han determinado que, en el siglo XIX, la población nacional se encontraba distribuida en la siguiente forma: $86 \%$ en el Valle Central, $13 \%$ en Guanacaste y Pacífico Central, el 1 \% en el resto del país (Gómez Barrantes y Madrigal Pana, 2004, p. 519).

Pese a esta realidad inicial, ciertamente, los estudios de geografía lingüística del profesor Miguel Ángel Quesada Pacheco posteriores a 1992, han señalado la necesidad de precisar esta zonificación original para visibilizar otras regiones que han ido adquiriendo una configuración distintiva. En todo caso, esta intervención no busca llegar a zanjar esta discusión y aprovecha la información suministrada por el mismo Quesada, quien en todas las propuestas de atlas ha distinguido la región noroeste de la meseta central como zonas dialectales diferentes. 
Quesada Pacheco (2013) presenta una detallada exposición de las zonas dialectales costarricenses y llega a la siguiente conclusión general:

Son relativamente pocos los intentos que se han hecho en miras a proponer o describir una división dialectal de Costa Rica. Los autores que lo han intentado han sido H. Polakowski, L. Dobles Segreda, A. Agüero y M. A. Quesada Pacheco. Dichos intentos se pueden agrupar en dos etapas: menciones implícitas de una división dialectal, y menciones explícitas. La primera etapa va de 1876 a 1990; la segunda, de 1990 hasta la fecha actual (Quesada Pacheco, 2013, p. 44).

Tanto Polakowski, Segreda y Agüero, es decir, autores del primer período, concuerdan en diferenciar la zona del Pacífico del Valle Central; sin embargo, sorprendentemente, Quesada Pacheco (1992 y 2010) ${ }^{3}$-representante del segundo períodovuelve a presentar estas zonas diferenciadas entre sí, amén de las otras zonas que ha distinguido posteriormente. Es decir, existe una configuración constante en la zonificación dialectal costarricense en relación con esas dos regiones geográficas.

En definitiva, para los objetivos de esta investigación, basta subrayar la comprobada realidad de que estas dos variedades costarricenses muestran variación en los diferentes niveles de la lengua. En especial en el nivel léxico, las diferencias son notables y ni qué decir si se trata de fitónimos o de alimentos.

Por esa razón, este acercamiento a la gastronomía en relación con las diferencias dialectales, mostrará como la presencia o ausencia de algunas voces puede ayudar a perfilar mejor estas zonas, así como las relaciones que se pueden mantener entre ellas.

Para describir la zonificación dialectal del español de Costa Rica, sirve excelentemente la propuesta de Quesada Pacheco y Vargas Vargas en el 2010 -comentada ampliamente en el artículo "División dialectal de Costa Rica según sus hablantes” (2013), de Quesada Pacheco-, sin perder de vista su primera propuesta en 1992.

Interesa, sobre todo, resaltar que en ambas propuestas la zona noroeste del país se mantiene bien conservada como área lingüística homogénea, frente al avance que se muestra para la zona central en otras regiones del país, quizás por las ventajas socio-

\footnotetext{
3 Esta propuesta solo considera datos fonéticos.
} 
económicas que le aseguran una posición hegemónica. En definitiva, estas dos zonas tradicionalmente han tenido una presencia desde la Colonia; aunque sus posiciones hegemónicas entre ellas han sido fluctuantes. En la actualidad, la variante vallecentraleña goza de mayor aprecio y consideración como dialecto hegemónico.

Además, en el mapa presentado en el 2010, se evidencia la conexión existente entre la región guanacasteca y la zona sur. Por otra parte, llama la atención de la adscripción de las llanuras del Caribe a la zona central. En todo caso, es sintomático que, en una primera aproximación, se hubiera considerado como una región aparte. Por lo demás, es de sobra conocida la situación linguiística compleja de esta zona; en primer lugar, por el poblamiento tardío de la región y su incorporación como zona agrícola al resto del país que obligó a la masiva migración de habitantes de todas las regiones de Costa Rica; en segundo lugar, la presencia de variedades no hispánicas como el criollo limonense y la lenguas amerindias. Esta situación, aunada a la hegemonía vallecentraleña, explican su incorporación o acercamiento a la variedad del centro del país.

\subsection{Migraciones}

Los estudios demográficos en Costa Rica han demostrado que las migraciones han modificado la geografía humana, principalmente desde el siglo XIX; en primer lugar, por el agotamiento de la frontera agrícola y luego por los nuevos cultivos en zonas muy poco pobladas en Costa Rica. El tráfico de personas también ha impactado en las fronteras dialectales, pues los hablantes llevan consigo su acervo lingüístico propio, el cual pronto se adapta a las nuevas circunstancias de residencia. Los hechos lingüísticos conservados o exportados sirven como huellas indelebles de estos desplazamientos humanos. En esta forma, se pueden lograr comparaciones -en términos de distanciamiento o acercamientoentre los dialectos hispánicos de nuestro país.

Los estudios de Gómez Barrantes y Madrigal Pana (2004) han demostrado este vaivén poblacional y han señalado algunas constantes que lo han caracterizado. En la siguiente cita, es posible logar una idea aproximada de estos movimientos en el período 1927-2000:

Históricamente el proceso migratorio costarricense se ha caracterizado por un 
movimiento desde las áreas rurales del Valle Central hacia las zonas periféricas costeras y no costeras. Este proceso alcanzó su máxima intensidad en los años sesenta y luego moderó su dinámica; también hubo cambios de dirección, ya que emergieron corrientes migratorias de las zonas periféricas hacia el Valle Central. Como resultado, la parte de la provincia de San José ubicada en el Valle Central ha mantenido una participación prácticamente estable dentro de la población nacional, el resto del Valle la ha visto disminuida marcadamente y el resto del país la ha aumentado significativamente. [...] A nivel de provincia se tiene que en el período 1927-2000 solamente Limón ha sido siempre de atracción y sólo Guanacaste de expulsión; el resto de las provincias muestra períodos en que predominó la inmigración y otros en que lo hizo la emigración (Gómez Barrantes y Madrigal Pana, 2004, p. 519).

Es menester destacar el papel desempeñado por el Valle Central como centro hegemónico lo que provoca que su dialecto se imponga sobre otras variedades tanto hispánicas como no hispánicas. A la vez, es muy insoslayable destacar que Guanacaste ha sido una región de expulsión, con lo que su variedad dialectal ha llegado a otras regiones costarricenses, así como Limón ha sido zona de atracción. La documentación de la voz chicheme ilustra perfectamente estos desplazamientos poblaciones, como se demostrará en el apartado 3.1.2 de este artículo.

Por estas razones, todo estudio comparativo de las zonas dialectales costarricenses debe recalar primero en la consideración de los movimientos demográficos, como se pretende visibilizar en este análisis de la geolingüística de la voz para referirse a una bebida tradicional del Noroeste del país.

\section{Correspondencias léxicas en el español de Costa Rica}

Echando mano a la noción de correspondencia, en este apartado, se examina la existencia de la palabra "chicheme" en varias zonas nacionales, que nos ayuda a comprender la base cultural que sustenta las zonas dialectales del Noroeste y sus zonas de influencia, así como las relaciones entre ellas promovidas, principalmente, por las migraciones. 
Para lograr este objetivo, la colección de libros titulada Cocina tradicional costarricense presta un valioso apoyo documental. Esta serie se publicó con el patrocinio del Ministerio de Cultura y Juventud, el Centro de Investigación y Conservación del Patrimonio Cultural, así como el Instituto Costarricense de Turismo. Estas obras nacen a partir de los certámenes de comidas y bebidas tradicionales, organizados por el Ministerio de Cultura y Juventud, con una amplia participación de la ciudadanía costarricense en todo el territorio nacional, desde el 2001 hasta el 2013. Todas las provincias están representadas, además se cuenta con recetarios para la zona norte y la zona sur. Las recetas fueron compiladas por la señora Yanory Álvarez Masís. Estas obras pueden ser consultadas en el sitio web www.visitecostarica.com.

La presentación por provincias y de dos regiones nos facilita el entrecruce de la información y registro léxicos y las zonas dialectales delimitadas, de manera que es fácil determinar la presencia o ausencia de los términos en estudio en las variedades lingüísticas allí utilizadas y sus conexiones. Asimismo, como se ofrecen las recetas, se puede constatar si las voces tienen referentes diversos o adaptados al ambiente cultural de las regiones.

\subsection{Chicheme}

El chicheme es una bebida refrescante; pero, antes de valorarla como correspondencia léxica y su dispersión en la geografía dialectal costarricense, es preciso dar más detalles sobre su documentación en obras lexicográficas, su adscripción geográfica original y sus posibles etimologías.

\subsubsection{Registros en instrumentos lexicográficos y otras obras}

\subsubsection{Obras lexicográficas costarricenses}

El rastreo de información se inició con la consulta de los principales instrumentos lexicográficos que recogen las voces consideradas costarriqueñismos. El primer objetivo es constatar su registro, su definición y marcas geográficas.

La primera obra en recoger este vocablo es el Diccionario de Costarriqueñismos de Carlos Gagini, publicado originalmente en 1919. Su entrada es la siguiente: "Chicheme.Refresco de maíz cocido (pero sin moler) con leche y azúcar. Se usa en México y C. A. 
Chichen en el habla de los mozárabes es cocer, sancochar (Simonet. Glos. De voces moz.)” (Gagini, 2008, p. 64).

De esta entrada, vale destacar que no se menciona un lugar de uso específico para Costa Rica, se hace referencia tan solo a que es de uso en Mesoamérica. También se ofrece la etimología del vocablo, de la cual se sugiere el parentesco con la palabra mozárabe chichen. ${ }^{4}$ En fin, en cuanto a la etimología todavía podría considerarse incierta por la cantidad de posibles orígenes postulados. Pero, de más trascendencia para este estudio, es la preparación de la bebida en sí; pues, dista mucho de la preparación actual en la región noroeste del país, zona a la cual está asociada hoy esta palabra casi con exclusividad; por el contrario, dicha preparación se acerca más a las documentadas en otras zonas del país, por ejemplo en Limón o la Zona Sur, o fuera del país en Panamá.

En la entrada correspondiente a "pujagua”, Gagini (2008) ofrece más datos sobre el chicheme, pues se consigna que, con esa clase de maíz morado, se prepara el chicheme, el pozol y el atol; además se informa de que la voz es de uso en Guanacaste, con lo cual en forma indirecta se relacionan estas bebidas con la gastronomía de la zona. Hubiera sido deseable incluir esta información en la entrada correspondiente a chicheme para completar una mejor descripción de la bebida.

En progresión cronológica, aparece en 1991, el Nuevo diccionario de costarriqueñismos a cargo de Miguel Ángel Quesada Pacheco. Esta obra le dedica una entrada a la voz en cuestión, en los siguientes términos: “chicheme m. (Guanacaste, Puntarenas) Bebida hecha a base de maíz pujagua, la cual se va fermentando paulatinamente" (Quesada Pacheco, 2007, p. 115).

Con esta obra, se asocia explícitamente el nombre de la bebida con un espacio geográfico nacional que, a la vez, corresponde más o menos a la configuración espacial del dialecto del Noroeste del país. Además, se menciona que la base de esta receta es el maíz pujagua, el cual es el más usado en nuestro país para elaborar la bebida. En ese mismo año, Quesada Pacheco publica un libro sobre el español guanacasteco, en cuyo glosario corrobora el uso de esta voz en esa variedad (1991, p. 132).

\footnotetext{
${ }^{4}$ En consultas en Internet, se sugiere un parentesco con chicha, voz de origen kuna, y aún se le considera voz perteneciente al náhuatl; sin embargo, estas referencias son poco confiables pues las fuentes donde se registran no son consideradas académicamente sólidas.
} 
Pasados cinco años, en 1996, la Asamblea Legislativa publica el Diccionario de costarriqueñismos de Arturo Agüero Chaves. Esta obra publicada tardíamente contiene información recopilada por su autor muchos años antes. Define chicheme en la siguiente forma: “chicheme. m. Guan. Refresco de maíz morado, llamado pujagua, cocido y un poco fermentado" (Agüero Chaves, 1996, p. 94).

En este artículo, se restringe el uso de la voz a Guanacaste y su descripción se relaciona estrechamente al uso del maíz pujagua. Esta definición y la de Quesada Pacheco muestran una gran coincidencia y retratan muy de cerca el uso actual, así como indican dos regiones donde actualmente se utiliza esta palabra. No obstante, gracias al examen de los recetarios de comidas tradicionales - referidos en el apartado 3-, hoy se sabe que esta voz ha trascendido esas fronteras geográficas y se ha proyectado a otras regiones del país.

Por su parte, Luis Ferrero Acosta presenta una obra titulada Mil y tantos tiquismos, costarricensismos, en 2002. En ella, registra el término de interés de esta manera:

Chicheme. (En el habla de los mozárabes chichen es cocer, sancochar) m. Bebida hecha de maíz pujagua sazón, dulce, olores, jengibre y agua. Se fermenta paulatinamente. Se pone a remojar el maíz morado un buen rato y luego se le muele fino. La masa se cuece y se le agrega agua fría y se cuela la masa (Ferrero Acosta, 2002, p. 69).

Como se puede apreciar, este registro agrega poca información a la ya ofrecida. Su aporte es más bien de corte enciclopédico. Se trata de un resumen de la definición de Gagini en lo etimológico y, en cuanto al contenido, muestra poca diferencia con las definiciones de Agüero y Quesada.

Finalmente, resultó imperioso corroborar estos datos en alguna obra regional específica del español guanacasteco para recabar información específica de la zona. Por ello, se consultó el Diccionario de guancastequismos (2001), el cual presenta la siguiente definición: "chicheme: bebida hecha de maíz pujagua, con jengibre y dulce” (Gardela, 2001, p. 80). 
En cuanto a información sobre la bebida en sí, esta obra no resultó relevante, pues se apunta a la receta base registrada en los recetarios más adelante comentados, propia de Guanacaste y Puntarenas. Pero, el afán de Gardela en rescatar datos de la identidad cultural de la población guanacasteca con este diccionario, permitió conocer información relevante de esta bebida, como el tipo de actividades comunales con las que se asocia. En primer lugar es importante destacar que el chicheme se asocia mucho con las actividades de carácter religioso como rezos, bautizos, matrimonios, Semana Santa, culto al Cristo de Esquipulas o las fiestas dedicadas a la Virgen de Guadalupe, en las cuales son las jarreras y los diputados ${ }^{5}$ quienes se encargan de preparar y repartir la bebida. Por otra parte, se registra la siguiente unidad fraseológica: ya está cogiendo punto el chicheme y el tiste levantando copete para referirse a que ha alcanzado la plenitud una acción. Con ello, se demuestra que la palabra en estudio goza de gran vitalidad al punto de crear su propia fraseología. Por último, en esta obra se menciona otra bebida parecida al chicheme con la diferencia que se prepara con maíz blanco que recibe el nombre de cotón. Esta variante para preparar la bebida se encuentra en otras zonas del país. Finalmente, queda claro que el chicheme es una bebida integrada a la cultura guanacasteca y esta palabra está fuertemente anclada en el repertorio léxico de esa variedad dialectal del país.

\subsubsection{Otras obras lexicográficas}

La Real Academia Española entregó, en 2010, el tan esperado Diccionario de americanismos, con el patrocinio de la Asociación de Academias de la Lengua Española; esto significa que se cuenta con el examen y aprobación de las academias americanas correspondientes. A la letra, se registra así la voz chicheme:

\section{chicheme}

I. 1. m. CR:NO. Bebida refrescante elaborada con maíz pujagua cocinado y molido, panela y jengibre.

2. $\quad$ m. $P a$. Bebida popular hecha del agua de cocción del maíz pilado y del propio grano, a la que se le agrega leche, miel de caña

\footnotetext{
5 Según Gardela (2001), en su Diccionario de guanacastequismos, son los nombres con los que son llamadas las mujeres y hombres que se encargan de hacer y repartir el chicheme en esa fiesta religiosa.
} 
de azúcar y canela o vainilla (Asociación de Academias de la Lengua Española, 2010, p. 513).

En este artículo, la descripción de contenido no se aparta mucho de las definiciones encontradas en otras obras lexicográficas para Costa Rica. En el caso de Panamá, el contenido de la definición cambia. Ahora bien, resulta interesante que la voz solo se registre para estos dos países. Esta asignación geográfica coincide con las búsquedas realizadas en diccionarios de regionalismos para el área centroamericana, pues estos no la registran. El Diccionario de americanismos de la RAE es una voz autorizada para circunscribir su uso solo a estas dos naciones, ya que todas las academias americanas fueron consultadas sobre el uso de los términos incluidos en dicho diccionario. Quizás sea prudente no afirmar categóricamente su inexistencia en otras variedades hispanoamericanas, pero sí cabe la posibilidad de que el término no sea de relevancia para otras comunidades de habla.

En consonancia con los hallazgos en la obra de la Real Academia Española, la obra de Baltasar Isaza Calderón, titulada Panameñismos, registra esta voz, en la siguiente forma: “CHICHEME m. Bebida preparada con maíz sin moler, que se hierve en agua y se adiciona con leche, azúcar y algún otro ingrediente” (Isaza Calderón, 1963, p. 188).

La descripción de esta receta, como veremos más adelante, coincide con la preparación documentada en la zona sur de Costa Rica.

\subsubsection{Tesis}

En 2010, la investigadora Solveig Hjelleset defiende su tesis de maestría, cuyo título es El léxico de la comida en Guatemala. Análisis geolingüístico. Esta obra confirma el uso preferente en Costa Rica y Panamá, pues registra la voz chicheme como forma compartida en las variedades de ambos países. Además, también afirma que Nicaragua comparte el uso, aunque con la consulta de autores como Mantica (1989) sobre el español de este último país no se logró confirmar dicho uso:

Por otro lado, hay palabras compartidas entre dos o tres países, como taco

(Guatemala y Honduras), rigua (Guatemala, El Salvador y Honduras), 
mazamorra y chicheme (Costa Rica y Panamá), buñuelo/biñuelo/boñuelo (Nicaragua, Costa Rica y Panamá), nacatamal (Honduras, El Salvador, Nicaragua), yoltamal/yoles (Nicaragua y Costa Rica), pupusa (El Salvador y Honduras), atol chuco (Guatemala, Honduras y El Salvador), indio viejo en Nicaragua e indio pelado en Costa Rica (Hjelleset, 2010, p. 76).

Nicaragua y Costa Rica también comparten algunas palabras; yoltamal, tamal mudo, vigorón, aguachacha, mazamorra, lengua (mapa 28), enyucado, chicheme, tanela, yoltamal, pujagua, cena, tamal dulce. Por último, se observan palabras compartidas entre Costa Rica y Panamá, como chicheme, mazamorra, tamal de olla, guacho, patacon(es) (Hjelleset, 2010, p. 79).

En síntesis, de esta investigación no se logra determinar la presencia de la voz chimeme en Guatemala, ni se menciona para otros países centroamericanos, excepto Nicaragua. Por otra parte, se subraya el uso compartido entre Costa Rica y Panamá, hecho confirmado con la presente investigación en recetarios de estos dos últimos países. Pese a no haber sido posible la documentación de la palabra en obras autorizadas del dialecto costarricense, y dándole crédito a la información de Hjelleset, se tendría una valiosa información que apuntaría a un uso compartido en una macro zona dialectal que recorrería las costas de estas tres naciones centroamericanas.

\subsubsection{Tipos de chicheme y su geolingüística}

En el 2005, el Ministerio de Cultura, Juventud y Deportes (Álvarez Masís, 2005) publica la primera obra sobre recetas tradicionales que corresponde a la provincia de Guanacaste y a la región central de Puntarenas. Estas localidades, además, coincidentemente integran una misma zona dialectal en las propuestas geolinguiísticas de Quesada Pacheco, presentadas anteriormente.

En dicho compendio, aparecen dos recetas de chicheme para Guanacaste y cuatro para Puntarenas, aunque en el concurso solo dos formas de preparar la bebida fueron 
seleccionadas como ganadoras (Álvarez Masís, 2005, pp. 76, 145-146). En lo fundamental, ambas recetas utilizan como base de preparación: maíz pujagua, tapa de dulce y jengibre. Cabe resaltar que, en esta zona noroeste del país, la bebida se preparar únicamente con maíz pujagua. Otros ingredientes que se utilizan optativamente son el clavo de olor, la canela y la vainilla. Además, se puede sustituir la tapa de dulce por azúcar procesada. Su preparación es muy elaborada, pues requiere utensilios particulares, como tinajas curadas y piezas de una manta especial como colador; sin embargo, en algunas recetas se simplifica su elaboración, por ejemplo, usando baldes plásticos o pascones industrializados. Debido a que es una bebida fermentada, se necesitan varios días para lograr el punto agradable al paladar.

El segundo hallazgo de chicheme como bebida tradicional se realizó en el recetario que corresponde a la provincia de Limón, publicado por el mismo ministerio en 2014 (Álvarez Masís, 2014). En este caso, en el concurso gastronómico se seleccionaron dos recetas; una de ellas coincide con la preparación usual en el noroeste del país. En cambio, en la segunda, figuran nuevos ingredientes: leche regular y leche condensada; además, se le agrega harina de trigo y leche de coco (Álvarez Masís, 2014, p. 41). Este último ingrediente sugiere la adaptación de la bebida a la cultura limonense. Este rasgo de aculturación se evidencia aún más, pues recibe también el nombre "homminy", proveniente del criollo limonense. Por otra parte, en la receta no se menciona el uso específico de maíz pujagua, tan solo se menciona que se usa maíz cascado. El resultado es muy diferente de la versión guanacasteco-puntarenense, pues aquí se logra una especie de atol.

Es significativa la documentación de esta voz dentro de las costumbres alimentarias de la provincia de Limón. En primer lugar, se sabe que esta zona del país experimentó una fuerte migración de población del noroeste del país a causa del cultivo de banano, en consonancia con la tradicional característica de esta zona, como lugar de atracción demográfica (Gómez Barrantes: 2002, p.500). Además, el nombre en criollo limonense apunta hacia la situación lingüística particular de la región.

El tercer hallazgo se obtuvo en el recetario dedicado a la Zona Sur del país (Badilla Chang, 2015). En él, se registran doce diferentes recetas, las cuales van desde la receta básica encontrada en Guanacaste y Puntarenas, hasta las preparaciones encontradas en Limón. La mayoría incluyen el uso de maíz pujagua, mas algunas se preparan con maíz 
amarillo. Como en el caso limonense, algunas mezclas incluyen los productos lácteos, con lo cual se establece un nexo no solo con esa provincia costarricense sino con bebidas panameñas que luego se presentarán. Asimismo, una diferencia con el noroeste del país donde se usa exclusivamente el maíz pujagua o morado y no se agregan productos lácteos (Badilla Chang, 2015, pp. 121-125).

Con respecto a las tradiciones regionales y las comidas llegadas a la Zona Sur, como consecuencia de las migraciones, es interesante citar en este momento de Marjorie Ross (2001):

La composición de la población era muy heterogénea, integrada en su mayoría por personas provenientes de Honduras, Nicaragua, Puntarenas centro y el interior del país, por lo que no existe allí una cocina original, sino que se trata de platos traídos por los inmigrantes, que vinieron para quedarse. Sin embargo, dentro de esa heterogeneidad, hay un predominio muy marcado de la cocina nicaragüense. Desde los primeros años las fondas en los mercados eran propiedad de nicaragüenses, y eran nicas también las vendedoras de comida que se apostaban en distintos lugares. También a través de la cocina puntarenense, influida, a su vez, por la cuchara nicaragüense, se colaban las recetas nicas. Es importante destacar este hecho, que permite ver que la influencia culinaria del hermano país se ha extendido por un período de tiempo bien largo, desde el norte hasta el sur de nuestro litoral pacífico (Ross González, 2001, p. 190).

En general, es muy acertado destacar esta coincidencia de costumbres alimentarias en la región pacífica; no obstante, no se debe considerar en términos de influencia o superposición, sino más bien en rasgos culturales compartidos en una macrozona; situación que se evidencia en la difusión de la lengua castellana por estas regiones. Asimismo, es importante señalar que la abundancia de recetas para el chicheme en esta zona refleja la composición heterogénea de sus pobladores en cuanto a su procedencia.

Es sintomático que, en los recetarios de las provincias de San José, AlajuelaHeredia, Cartago y Zona Norte -también publicados por el Ministerio de Cultura, Juventud y Deportes en los años 2013, 2013, 2010 y 2014 respectivamente, no aparezca esta bebida a 
base de maíz. Definitivamente así se marca que esta voz y su referente no pertenecen a su geolecto ni a su tradición culinaria vallecentraleña. Al respecto, Quesada Pacheco (1991), en su libro Español de Guanacaste, afirma:

Aún más significativo es el grupo de palabras usadas en Guanacaste, las cuales aparecen registradas en varios países de América pero se desconocen en el Valle Central, entre las que se citan aguada, atolada, barajustar, berrinche (con el sentido de 'mal olor'), canjuro, cayuco, cojinuda, curcucho, chapín, chicheme, chirizo, fierra, hostigoso, maneto, matacán, montuno, ñanjú, prestar ('pedir prestado'), requeneto, sorococa, turún, yerrugate. Sin duda, estas palabras dan cuenta del contacto que hubo entre el Partido de Nicoya y otras regiones del continente americano durante la Colonia (p. 100, destacado añadido).

Dada la sugerencia de la cita anterior, se procedió a investigar la presencia de esta voz para otras zonas americanas. El primer hallazgo se encontró en el país vecino del Sur: Panamá; en el diccionario titulado Panameñismos (Isaza Calderón, 1963). Además, en fuentes de Internet, hay abundantes referencias a esta bebida la cual se asemeja más a un atol que es consumido como postre. Gracias a esta evidencia, se vuelve a confirmar que esta voz pertenece a una macrozona cultural y lingüísticas que recorre el litoral centroamericano, cuya formación se remontaría al período de conquista y poblamiento por parte los expedicionarios españoles del siglo XVI, en franca distancia con la tradición dialectal fundante de la variedad vallecentraleña.

Tal parece que es muy famoso el chicheme de la región de la Chorrera, constituye una bebida típica de la zona. Para su preparación se usa maíz amarillo y, a diferencia del guanacasteco, se le agrega leche y maicena para espesar, con lo cual adquiere una consistencia de atol. El uso de lácteos se documenta en Limón y la Zona Sur; sin embargo, en ninguna de las recetas costarricenses se utiliza maicena; en Limón, se usa harina para espesar. Esta versión caribeña costarricense es la que más se aproxima al panameño. Estas modificaciones o variaciones en la forma de preparación se pueden explicar fácilmente, teniendo en cuenta las migraciones entre estas zonas, que provocan mezclas de tradiciones culturales y lingüísticas. 


\section{Conclusiones}

Como se ha mencionado, la variación léxica depende altamente de los factores extralingüísticos, como los que han intervenido en este caso, a saber: migraciones, adaptaciones a la realidad geográfica y cultural de las comunidades. En el campo del léxico gastronómico, esta influencia es definitiva; al variar los productos de la tierra se utilizan voces asociadas a otros pero que guardan algún grado de semejanza.

El ejemplo examinado puede ser considerado una correspondencia léxica. Esta forma de variación léxica está documentada ampliamente en la formación del español americano desde los primeros tiempos de la exploración, conquista y poblamiento de América por España.

En la geografía dialectal costarricense, la voz chicheme se asocia definitivamente con la variedad utilizada en el noroeste del país y con la base cultural de esa región. Esta voz se ha difundido por otras regiones donde la población guanacasteca o puntarenense ha ejercido su influencia gastronómica; por ello, se pudo atestiguar también su uso en la zona caribeña y sureña. Queda excluido de su radio de propagación el centro del país, por pertenecer a otra tradición cultural y dialectal. Esta tarea fue posible gracias a las obras especializadas sobre la cocina tradicional costarricense, auspiciadas por el Ministerio de Cultura y Juventud.

Los registros en las obras lexicográficas confirman la distribución interna en la geografía nacional, así como su dispersión en la región mesoamericana, especialmente por Costa Rica y Panamá. Queda pendiente ampliar esta revisión a obras de disciplinas relacionadas, porque es presumible que se encuentre en otras variedades linguiísticas centroamericanas, pese a que el Diccionario de Americanismos de la Asociación de Academias de Lengua Española (2010) y la tesis de Solveig Hjelleset (2010) restrinjan su uso a estos dos países. El expurgo de los diccionarios regionales tampoco arrojó luces para determinar con certeza la etimología; por ello, se requieren nuevas pesquisas bibliográficas.

Por último, este estudio puede ampliarse a otras voces que muestran el mismo comportamiento, tales como: pozol y bizcocho, las cuales aunque presentes tanto en el noroeste como en el centro del país no apuntan a los mismos contenidos semánticos. También puede ser una veta para conocer la diferenciación dialectal del español hablado en 
Costa Rica el examen del caso de gallo pinto en el Valle Central y burra en la zona sur. En fin, es una invitación a profundizar los estudios de dialectometría en Costa Rica y zonas lingüísticas aledañas.

\section{Referencias bibliográficas}

Agüero Chaves, A. (1996). Diccionario de costarriqueñismos. San José: Asamblea Legislativa.

Álvarez Masís, Y. (2005). Cocina tradicionala costarricense 1: Guanacaste y Región Central de Puntaremas (C. d. nacional, Ed.) San José.

Álvarez Masís, Y. (2010). Cocina tradicional costarricense 3: Cartago. San José: Centro de investigación y conservación del patrimonio cultural.

Álvarez Masís, Y. (2013). Cocinal tradicional costarricense 4: Alajuela y Heredia. San José: Centro de investigación y conservación del patrimonio cultural.

Álvarez Masís, Y. (2013). Cocina tradicional costarricense 5: San José. San José: Centro de investigación y conservación del patrimonio cultural.

Álvarez Masís, Y. (2014). Cocina tradicional costarricense 6: Limón. San José: Centro de investigación y conservación del patrimonio cultural.

Asociación de Academias de la Lengua Española. (2010). Diccionario de americanismo. Madrid: Santillana.

Badilla Chang, I. (2015). Cocina tradicional costarricense 8. Zona Sur: Osa, Corredores y Golfito. San José: Cetnro de investigacción y conservación del patrimonio cultural.

Ferrero Acosta, L. (2002). Mil y tantos tiquismo, costarricensismos. San José: EUNED. 
Gagini, C. (2008). Diccionario de costarriqueñismos (4. ${ }^{a}$ ed.). San José: Editorial Costa Rica.

Gardela, M. T. (2001). Diccionario de guanacastquismos. San José: ICER.

Gómez Barrantes, M. y Madrigal Pana, J. (2004). Migración interna en Costa Rica en el período 1927-2000. En L. Rosero Bixby, Costa Rica a la luz del censo del 2000 (p. 594). San José: Centro Centroamericano de Población (CCP), UCR.

Hjelleset, S. (2010). El léxico de la comida en Guatemala (Tesis de maestría). Universidad de Bergen, Bergen.

Isaza Calderón, B. (1963). Panameñismos. Panamá: Publicaciones de la Academia Panameña de la Lengua.

Mántica Abaunza, C. (1989). El habla nicaragüense y otros ensayos. San José: Asociación Libro Libre.

Moreno Fenández, F. (1998). Principios de sociolingüística y sociología del lenguaje. Barcelona: Editorial Ariel S. A.

Quesada Pacheco, M. (1991). El español de Guanacaste. San José: Editorial de la Universidad de Costa Rica.

Quesada Pacheco, M. (2007). Nuevo diccionario de costarriqueñismos. Cartago: Editorial Tecnológica de Costa Rica.

Quesada Pacheco, M. (2013). División dialectal de Costa Rica según sus hablantes. Dialectologia et Geolinguistica. Jounal of the International Society for Dialectology and Geolinguistics, 36-69. 
Ross González, M. (2001). Entre el comal y la olla. San José: EUNED.

Urbina Gaitán, R. (2014). Cocina tradicional costarricense 7: Zona Norte. San José: Centro de investigación y conservación del patrimonio cultural. 